\author{
22-24 November, 2019 \\ Paris, France
}

\title{
Frequency Analysis of Vibrations of Composite Sandwich Beams with Viscoelastic Core
}

\author{
Khadri Youcef ${ }^{1}$, Tekili Sabiha², Karmi Yacine ${ }^{3}$, Daya El Mostafa ${ }^{4}$, Daouadji Ali ${ }^{5}$ \\ ${ }^{1}$ Laboratoire de Mécanique Industrielle, Université Badji Mokhtar BP 12 Annaba, Algérie \\ ${ }^{2}$ Université de Lorraine, Laboratoire d'Etude des Microstructures et de Mécanique des Matériaux \\ (LEM3), UMR CNRS 7239,7 rue Félix Savart BP 15082 F-57073 Metz Cedex 03, France \\ ${ }^{3}$ Laboratoire GEOMAS INSA-Lyon, Bâtiment J.C.A. Coulomb - 34 avenue des Arts \\ 69621 Villeurbanne Cedex, Lyon, France
}

\begin{abstract}
The present study is concerned with the dynamic analysis of sandwich beams, with a viscoelastic core between composite layers and subjected to a moving load. The governing equations are derived by application of Hamilton principle and solved by finite element method. The coupled system of equations is integrated numerically by the Newmark method. Facial composites are modeled using classical plate theory and the kernel using Timoshenko's theory. This study deals with free and forced vibrations of the sandwich beams by considering the frequency according to the viscoelastic properties in frequency- domain and the numerical asymptotic method is employed in order to solve the complex eigenvalue problem. The influences of viscoelastic loss factor, orientation fiber of the composite layers and thickness ratio on the dynamic responses of sandwich beams are investigated. The results reported in this paper show that the viscoelastic loss factor has a significant effect on the vibration behavior involving the improvement of the damping of the structure. The influence of fiber orientation on vibration behavior showed that the damping of the structure could be improved by adopting a better configuration of the composite layers. The results obtained with the effect of the thickness ratio show that the sandwich structure has a greater dissipative vibratory energy capacity for the low viscoelastic thickness values.
\end{abstract}

Keywords: vibration; sandwich; viscoelastic; composite; loss factor. 